\title{
COMPOSTOS BIOATIVOS DE EXTRATOS DE PEQUI DE DIFERENTES REGIÕES DO CERRADO
}

Camila Fernanda Dias de Oliveira ${ }^{1}$; Ellen Godinho Pinto ${ }^{2}$; Priscila Lidia Rosa de Rezende ${ }^{3}$

${ }^{1}$ Mestre em Ciência e Tecnologia de Alimentos da Universidade Federal de Goiás, Goiânia, Brasil (camilaferdias@gmail.com)

${ }^{2}$ Professora Mestre do Departamento de Tecnologia de Alimentos do Instituto Federal Goiano Campus Morrinhos, Morrinhos, Brasil.

${ }^{3}$ Tecnóloga em Alimentos do Instituto Federal Goiano Campus Morrinhos, Morrinhos, Brasil.

Recebido em: 08/04/2017 - Aprovado em: 10/06/2017 - Publicado em: 20/06/2017 DOI: 10.18677/EnciBio_2017A159

O pequi (Caryocar brasiliense Camp) é um fruto de elevado valor nutritivo, rico em substâncias com capacidade de sequestrar radicais livres, de grande importância para o bom funcionamento do organismo de forma geral, como por exemplo carotenoides, compostos fenólicos e vitamina C. O objetivo do trabalho foi caracterizar nutricionalmente a polpa de pequi e avaliar a extração aquosa e alcoólica de pequi, por meio das medidas biométricas, físico-químicas, teores de compostos fenólicos totais e carotenoides totais das regiões de Goiás, Minas Gerais e Tocantins. As características biométricas foram as que mais apresentaram variabilidade, constatando variações para massa da casca sendo que os frutos de Tocantins maior rendimento. Nas análises físico-químicas da polpa as maiores diferenças foram a vitamina $C$, que para Minas Gerais destacou. Para os extratos aquosos e alcoólicos das análises físico-químicas houve poucas variações no tempo, temperatura e regiões. Porém para $0{ }^{\circ}$ Brix os resultados foram bem flutuantes. Na temperatura $25{ }^{\circ} \mathrm{C}$ no tempo $12 \mathrm{~h}$ o extrato de pequi para região de Minas Gerais mostrou maior concentração de carotenoides totais na extração aquosa e alcoólica. Já para os compostos fenólicos a temperatura de $65^{\circ} \mathrm{C}$ a $24 \mathrm{~h}$ foi melhor o extrato aquoso de pequi em Goiás. E para extração alcoólica do pequi na temperatura $45^{\circ} \mathrm{C}$ a $1 \mathrm{~h}$ na região do Tocantins mostrando maior quantidade. Podese concluir que as características biométricas e químicas do pequi e dos extratos aquosos e alcoólicos, são bastante influenciadas pela região dos frutos mostrando a riqueza em compostos bioativos.

PALAVRAS-CHAVE: Carotenoides totais. Fenólicos totais. Superfície resposta.

\section{BIOACTIVE COMPOUNDS EXTRACTS PEQUI DIFFERENT REGIONS CERRADO}

\footnotetext{
ABSTRACT

The pequi (Caryocar brasiliense Camp) is a fruit of high nutritional value, rich in substances capable of sequestering free radicals, of great importance for the good functioning of the body in general, such as carotenoids, phenolic compounds and ENCICLOPÉDIA BIOSFERA, Centro Científico Conhecer - Goiânia, v.14 n.25; p. 17992017
} 
vitamin C. O The objective of this work was to characterize the pequi pulp and to evaluate the aqueous and alcoholic extraction of pequi through biometric, physicochemical, total phenolic and total carotenoid contents of Goiás, Minas Gerais and Tocantins regions. The biometric characteristics were the ones that presented the most variability, evidencing variations for the bark mass and the fruits of Tocantins greater yield. In the physical-chemical analysis of the pulp the major differences were vitamin $\mathrm{C}$, which for Minas Gerais highlighted. For the aqueous and alcoholic extracts of the physicochemical analyzes there were few variations in time, temperature and regions. However, for ${ }^{\circ}$ Brix the results were very fluctuating. At temperature $25^{\circ} \mathrm{C}$ in time $12 \mathrm{~h}$ the pequi extract for the region of Minas Gerais showed higher concentration of total carotenoids in the aqueous and alcoholic extraction. For the phenolic compounds at $65^{\circ} \mathrm{C}$ to $24 \mathrm{~h}$, the aqueous extract of pequi in Goiás was better, and for the alcoholic extraction of pequi at a temperature of $45^{\circ} \mathrm{C}$ to $1 \mathrm{~h}$ in the Tocantins region, showing a larger quantity. It can be concluded that the biometric and chemical characteristics of pequi and the aqueous and alcoholic extracts are strongly influenced by the fruit region showing the richness in bioactive compounds.

KEYWORDS: Surface response. Total carotenoid. Total phenolics.

\section{INTRODUÇÃO}

O cerrado é o segundo maior bioma nacional, localizado em quase tonalidade no planalto central que compõem os estados de Maranhão, Piauí, Pará, Mato Grosso, Goiás, Minas Gerais e Tocantins. É considerada uma das principais áreas para conservação da biodiversidade mundial e a mais rica flora dentre as savanas do mundo. Assim, a flora do cerrado possui diversas espécies frutíferas, cujos frutos se destacam por suas agradáveis peculiaridades exóticas, embora ainda sejam pouco explorados cientificamente e comercialmente (BEUCHLEA et al., 2015; RESENDE-MOREIRA et al., 2017).

Dentre os diversos frutos nativos do cerrado, destaca-se o pequi (Caryocar brasiliense Camp.) conhecido popularmente como piqui, pequiá, amêndoa de espinho, grão de cavalo ou amêndoa do Brasil. Estes merecem atenção especial, por sua elevada ocorrência no cerrado e pelas características de sua polpa. $O$ grande período de oferta de frutos de pequi, aliado à heterogeneidade das regiões produtoras, levam a crer na existência de diferenças entre suas características físicas e químicas (MACHADO et al., 2013; TRAESEL et al., 2016).

$O$ pequi é um fruto de elevado valor nutritivo, rico em vitaminas $A, E$, ácidos graxos, fósforos e magnésio. Além disso, são fontes de substâncias com capacidade de sequestrar radicais livres, de grande importância para o bom funcionamento do organismo de forma geral, como por exemplo carotenoides, compostos fenólicos e vitamina C (MENDONÇA et al., 2017).

O elevado teor de compostos bioativos encontrado no pequi vem apresentando um crescente interesse de consumidores e da sociedade científica, devido à grande ênfase dada à atividade antioxidante como modo de ação contra doenças crônicas e degenerativas, incluindo o câncer. A vitamina $\mathrm{C}$ por exemplo é essencial para a saúde humana, visto que está envolvida na formação de tecido conjuntivo, anticorpos, produção de hormônios e proteção antioxidante. Outras substâncias como os compostos fenólicos e carotenoides também estão associados no potencial antioxidante protegendo dos riscos de doenças cardiovasculares e podem atuar sobre o estresse oxidativo, relacionado com diversas patologias crônico-degenerativas. Além da influência no valor nutricional e na qualidade 
sensorial, conferindo em atributos como cor, textura, amargor e adstringência (PESSOA et al., 2015).

Portanto, diante da importância dos compostos bioativos e de sua atividade antioxidante para a saúde, assim como da biodiversidade do bioma cerrado, é relevante pesquisas que considerem as diferenças físicas, químicas e bioquímicas de frutos oriundos de diferentes regiões do cerrado. Neste contexto, o objetivo deste estudo foi a investigação de compostos bioativos, das medidas biométricas e propriedades físico-químicas da polpa e dos extratos aquosos e alcoólicos de pequi oriundos das regiões de Goiás, Minas Gerais e Tocantins.

\section{MATERIAL E MÉTODOS}

Foram adquiridos os frutos de pequi (Caryocar brasiliense Camp) nativos de três estados do bioma cerrado: Goiás, Minas Gerais e Tocantins. Logo após a aquisição foram selecionados, descartando-se os que apresentavam injúrias. Posteriormente foram submetidos a uma lavagem por imersão em água e sanificados por 15 minutos em solução de 100 ppm de hipoclorito de sódio. Em seguida os frutos foram descascados e despolpados manualmente com auxílio de faca de aço inox e submetidos as determinações das medidas biométricas e físicas e químicas, realizadas no laboratório de físico-química do Instituto Federal Goiano Campus Morrinhos

As extrações aquosas e alcoólicas seguiram a metodologia proposta por LONGHINI et al. (2007) onde, as polpas foram homogeneizadas em liquidificador com água destilada, para extração aquosa na proporção de 1:3 (m/m) fruta/água e com álcool etílico $70 \%$ para extração alcoólica na proporção de $1: 3(\mathrm{~m} / \mathrm{m})$ fruta/álcool. As extrações foram mantidas no banho maria a 25,45 e $65{ }^{\circ} \mathrm{C}$ e retiradas alíquotas desta em 1, 12 e 24 horas. As extrações foram filtradas com papel filtro Whatman e realizadas as análises de $\mathrm{pH}$, acidez titulável total, sólidos solúveis ( ${ }^{\circ}$ Brix), vitamina $\mathrm{C}$, carotenoides totais e fenólicos totais.

Para as análises biométricas foram selecionados, aleatoriamente doze frutos de pequi. Analisando as seguintes variáveis: massa da casca, massa da polpa e massa total do caroço. Com auxílio da balança semi-analítica (Bel Engineering), o rendimento de polpa foi determinado pelo quociente da massa total dos frutos pela massa total da polpa e caroços de cada fruto, de acordo com metodologia proposta por MOURA et al., (2013).

As determinações físicas e químicas foram avaliadas perante teor de umidade em estufa a $105^{\circ} \mathrm{C}$ (Tecnal, TE-395, Piracicaba, Brasil); resíduo mineral fixo (cinzas) por incineração em mufla à $550{ }^{\circ} \mathrm{C}$ (EDG, Forno Economic, São Carlos, Brasil) pH pelo método potenciômetro previamente calibrado com soluções padrão (Mettler Toledo, Brasilia, Brasil) a acidez titulável total foi determinada por titulação com $\mathrm{NaOH}(0,1 \mathrm{~N})$ com indicador fenolftaleína e expressa em termos de g de ácido cítrico; o teor de sólidos solúveis totais foi realizado por medida direta em refratômetro (Mettler Toledo, Brasilia, Brasil) e a vitamina $\mathrm{C}$ foi quantificada na oxidação do ácido ascórbico pelo iodato de potássio. Todas as metodologias seguindo IAL (2008).

As determinações de carotenóides totais foram realizadas por meio da extração com álcool etilíco $70 \%$ e analisadas em espectrofotômetro (BEL photonics, S 2000 UV, Osasco, Brasil) a $450 \mathrm{~nm}$, segundo a metodologia de LEÃO et al. (2017). As análises foram feitas em triplicata e os resultados foram expressos de acordo com a equação 1. 


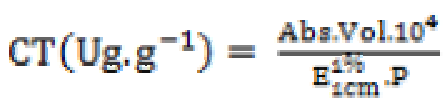

Eq. 1

Onde, $\mathrm{CT}=$ Carotenoides totais; Abs. = Absorbância no $\lambda$ máximo; Volume da diluição (mL); $\mathrm{E} 1 \% .1 \mathrm{~cm}=2592 ; \mathrm{P}=$ Peso da amostra $(\mathrm{g})$.

As determinações de compostos fenólicos Totais (CFT) seguiram a metodologia proposta por SOUSA et al., (2011), sendo a leitura da absorbância realizada em espectrofotômetro (BEL photonics, S 2000 UV, Osasco, Brasil) a $765 \mathrm{~nm}$ e os resultados expressos em mg equivalente de ácido gálico por grama de amostra (mgEqAE g ${ }^{-1}$ ) em base seca. A análise foi realizada em triplicata.

Para a comprovação das médias aritméticas, empregaram-se a análise de variância ANOVA e o para determinação das diferenças mínimas significativas foi utilizado o teste de Tukey ao nível de significância de $5 \%(p 0,05)$.

\section{RESULTADOS E DISCUSSÃO}

As características biométricas e físicas e químicas encontram-se nas Tabelas 1 e 2 , respectivamente. Pode-se observar que a massa da casca variou de $71,35 \%$ a $39,96 \%$, esses resultados indicam uma diferença significativa entre as médias, com superioridade de $71,35 \%$ para os pequis de Tocantins. De acordo com MOURA et al. (2013), altos valores de massa da casca indicam frutos grandes de baixo rendimento da polpa e, de certa forma, contribui para a oneração de transporte e até mesmo a inviabilização da industrialização.

TABELA 1. Características biométricas de pequi das regiões de Goiás, Minas Gerais e Tocantins.

\begin{tabular}{llcc}
\hline Característica & Goiás & Minas Gerais & Tocantins \\
\hline Massa da Casca\% & $48,46 \pm 0,21^{\mathrm{b}}$ & $39,96 \pm 0,20^{\mathrm{c}}$ & $71,35 \pm 0,10^{\mathrm{a}}$ \\
Rendimento de Polpa\% & $32,21 \pm 0,12^{\mathrm{b}}$ & $39,68 \pm 0,01^{\mathrm{a}}$ & $12,41 \pm 0,12^{\mathrm{c}}$ \\
Massa do Caroço\% & $19,31 \pm 0,11^{\mathrm{b}}$ & $20,31 \pm 0,03^{\mathrm{a}}$ & $16,23 \pm 0,03^{\mathrm{c}}$ \\
\hline
\end{tabular}

*Médias seguidas pela mesma letra nas linhas não diferem estatisticamente entre si, pelo teste Tukey, a nível de 5\% de significância.

Os resultados das médias das polpas de pequi mostraram que houve diferenças significativas entre as regiões. Os pequis com menor rendimento de polpa foram provenientes de Tocantins com média $12,41 \%$, seguidos dos de Goiás com $32,21 \%$, já os de Minas Gerais foram os de maior rendimento com 39,68\%.

Segundo VERA et al. (2005), quanto maior a quantidade de polpa maior a valorização econômica, pois é a parte do pequi mais consumida na alimentação humana e, por esse motivo, desperta maior interesse comercial.

Embora as massas dos caroços e de polpa de pequi de Tocantins foram menores. De acordo com VERA et al. (2007), tal fato faz com que o fruto tenha menor aceitação pelos consumidores e as indústrias processadoras de pequi. Isso porque, neste caso, é menor o rendimento na fabricação de conserva de polpa e molhos. Já o tamanho grande dos frutos dificulta o arranjo destes dentro da embalagem de vidro, resultando na desvalorização comercial desses frutos.

Por meio da observação dos valores das propriedades físico-químicas (Tabela 2) foi possível verificar que em termos gerais há uma variação nos valores analisados na polpa de pequi das regiões analisadas. 
TABELA 2. Composição físico-química da polpa de pequi provenientes dos estados de Goiás, Minas Gerais e Tocantins.

*Médias seguidas pela mesma letra nas linhas não diferem estatisticamente entre si,

\begin{tabular}{lccc}
\hline Constituintes & Goiás & Minas Gerais & Tocantins \\
\hline Umidade (\%) & $57,09 \pm 1,55^{\mathrm{a}}$ & $56,34 \pm 0,52^{\mathrm{a}}$ & $57,88 \pm 1,54^{\mathrm{a}}$ \\
Cinzas (\%) & $0,02 \pm 0^{\mathrm{a}}$ & $0,03 \pm 0^{\mathrm{a}}$ & $0,02 \pm 0^{\mathrm{a}}$ \\
ph & $5,74 \pm 0,25^{\mathrm{b}}$ & $6,02 \pm 0,02^{\mathrm{b}}$ & $7,21 \pm 0,10^{\mathrm{a}}$ \\
Acidez Titulável Total (\%) & $0,12^{\mathrm{a}}$ & $0,12^{\mathrm{a}}$ & $0,16^{\mathrm{b}}$ \\
$\begin{array}{l}\text { Sólidos Solúveis Totais } \\
\left({ }^{\circ} \text { Brix) }\right.\end{array}$ & $10,33 \pm 0,57^{\mathrm{ab}}$ & $9,66 \pm 0,57^{\mathrm{b}}$ & $11 \pm 0^{\mathrm{a}}$ \\
$\begin{array}{l}\text { Vitamina C (mg/100g de } \\
\text { ácido ascórbico) }\end{array}$ & $16,43 \pm 2,03^{\mathrm{c}}$ & $45,78 \pm 2,03^{\mathrm{a}}$ & $25,82 \pm 2,03^{\mathrm{b}}$ \\
\hline
\end{tabular}

pelo teste Tukey, a nível de $5 \%$ de significância.

Pode-se observar, na polpa de pequi apresentaram valor médio de umidade de $57,09 \%, 56,34 \%$ e $57,88 \%$ de Goiás, Minas Gerais e Tocantins, respectivamente. Resultados próximos para polpa de pequi, foram encontrados por Machado, Mello e HUBINGER (2015), relataram para região de Montes Claros - MG valores médios de $51,17 \%$. Por outro lado, SOUSA et al. (2012) em estudos com pequi proveniente do município de Crato - CE, observou valores médios de umidade maiores $82,58 \%$. O teor de umidade de um alimento está relacionado com sua estabilidade, qualidade e composição, já que elevados teores, podem afetar a estocagem e embalagem dos produtos, comprometendo sua qualidade (PEREIRA et al., 2003).

Para acidez titulável a polpa de pequi apresentou $0,12 \%$ e $0,16 \%$ de acidez, valores estes próximos dos encontrados por SOUSA et al. (2012), ou seja $0,3 \%$ de acidez titulável em polpa de pequi de Minas Gerais. Na polpa de Goiás e Minas Gerais não houve diferenças estatísticas, já a polpa de Tocantins diferiu das demais estatisticamente.

O teor de cinzas variou de $0,02 \%$ a $0,03 \%$, foi inferior aos relatados por OLIVEIRA et al. (2010) 0,63\% no estado do Piauí e LIMA et al. (2007) 0,6\% no estado do Ceará. Em geral em termos nutricionais, maiores teores de cinzas significam maiores quantidade de minerais (RAMOS \& SOUZA, 2011). Os resultados demonstraram que não houveram diferenças significativas nos valores de umidade $e$ cinzas.

$\mathrm{O}$ pequi, diferentemente da maioria das frutas tropicais, apresenta $\mathrm{pH}$ acima de 4,5, o que o classifica como fruto de baixa acidez (PAZ et al., 2014). Neste estudo o pH encontrado foi de 5,74, 6,02 e 7,21 nas polpas de Goiás, Minas Gerais e Tocantins respectivamente, valores superiores aos encontrados por SOUSA et al. (2012) de 4,5 nos frutos do município de Crato - CE. Sendo que os frutos de Tocantins apresentaram diferenças estatísticas dos frutos de Goiás e Minas Gerais. Porém esses valores foram próximos dos encontrados em frutos do cerrado como chichá 6,7 e macaúba 6,6 (ROCHA et al., 2011).

A polpa de pequi apresentou teores de sólidos solúveis totais de $10,33^{\circ}$ Brix, 9,66 ${ }^{\circ}$ Brix e $11^{\circ}$ Brix de Goiás, Minas Gerais e Tocantins, respetivamente. Valores estes superiores aos encontrados por SOUSA et al. (2012) de $4,5^{\circ}$ Brix nos frutos do município de Crato - CE. Ao mesmo tempo, mostrando-se diferenças estatísticas entre Minas gerais e Tocantins, porém não se diferem estatisticamente dos de 
Goiás. Com relação ao teor de sólidos solúveis totais, as concentrações podem ter variado conforme a intensidade da chuva durante o período de safra, a extensa época de oferta dos frutos, clima e características do solo (DANTAS et al., 2010; PAZ et al., 2014).

É possível verificar que o teor de vitamina $C$ das polpas de pequi de Goiás, Minas Gerais e Tocantins apresentaram diferenças estatisticamente significativas. Os valores foram $16,43 \mathrm{mg} / 100 \mathrm{~g}, 45,78 \mathrm{mg} / 100 \mathrm{~g}$ e $25,82 \mathrm{mg} / 100 \mathrm{~g}$, respectivamente. De acordo com MACHADO et al., (2015) em Montes Claros - MG a polpa de pequi possui $6,63 \mathrm{mg} / 100 \mathrm{~g}$ de vitamina C. Segundo SILVA \& NAVES (2001) a ingestão de frutas com teor de vitamina $C$ elevado é de grande importância, pois o ácido ascórbico apresenta atividade biológica, relacionando com a habilidade de neutralizar substâncias carcinogênicas pelo fato de possuir um potente poder antioxidante.

\section{Análises físico-químicas das extrações aquosas e alcoólicas do pequi}

Nas Tabelas 3 e 4 estão expressos os valores das análises físico-químicas das extrações aquosas e alcoólicas.

TABELA 3 - Resultados das análises físico-químicas das extrações aquosas do pequi das regiões de Goiás, Minas Gerais e Tocantins.

\begin{tabular}{|c|c|c|c|c|}
\hline \multicolumn{5}{|c|}{ Goiás } \\
\hline Temperatura & $\mathrm{pH}$ & $\begin{array}{l}\text { Acidez } \\
\text { Titulável Total }\end{array}$ & $\begin{array}{l}\text { Sólidos Solúveis } \\
{ }^{\circ} \mathrm{BRIX}\end{array}$ & Vitamina C \\
\hline $25^{\circ} \mathrm{C} /$ 1hora & $4,63 \pm 0,11^{b}$ & $0,14 \pm 0^{a}$ & $1,95 \pm 0,07^{b}$ & $6,5 \pm 2,49^{c}$ \\
\hline $45^{\circ} \mathrm{C} / 1$ hora & $4,80 \pm 0,04^{\mathrm{ab}}$ & $0,12 \pm 0,12^{\mathrm{a}}$ & $3,5 \pm 0^{\mathrm{a}}$ & $10,03 \pm 0,74^{b}$ \\
\hline $65^{\circ} \mathrm{C} / 1$ hora & $4,97 \pm 0,01^{a}$ & $0,11 \pm 0^{a}$ & $1,95 \pm 0,07^{b}$ & $26,61 \pm 0^{a}$ \\
\hline $25^{\circ} \mathrm{C} / 12$ horas & $4,31 \pm 0,41^{\mathrm{ab}}$ & $0,12 \pm 0^{a}$ & $1,3 \pm 0,14^{b}$ & $7,044 \pm 0^{c}$ \\
\hline $45^{\circ} \mathrm{C} / 12$ horas & $4,06 \pm 0,05^{b}$ & $0,13 \pm 0^{\mathrm{a}}$ & $3,5 \pm 0^{\mathrm{a}}$ & $10,21 \pm 0,49^{b}$ \\
\hline $65^{\circ} \mathrm{C} / 12$ horas & $5,29 \pm 0^{a}$ & $0,2 \pm 0,1^{b}$ & $3,5 \pm 0,14^{a}$ & $17,25 \pm 0,49^{a}$ \\
\hline $25^{\circ} \mathrm{C} / 24$ horas & $4,02 \pm 0,05^{b}$ & $0,22 \pm 0,12^{a}$ & $2,5 \pm 0^{c}$ & $10,56 \pm 0^{\mathrm{b}}$ \\
\hline $45^{\circ} \mathrm{C} / 24$ horas & $4,02 \pm 0,49^{b}$ & $0,12 \pm 0^{b}$ & $3,1 \pm 0,14^{b}$ & $7,04 \pm 0^{c}$ \\
\hline $65^{\circ} \mathrm{C} / 24$ horas & $5,26 \pm 0,02^{\mathrm{a}}$ & $0,16 \pm 0,3^{b}$ & $3,5 \pm 0^{\mathrm{a}}$ & $16,90 \pm 0,99^{\mathrm{a}}$ \\
\hline \multicolumn{5}{|c|}{ Minas Gerais } \\
\hline Temperatura & $\mathrm{pH}$ & $\begin{array}{c}\text { Acidez } \\
\text { Titulável Total }\end{array}$ & $\begin{array}{c}\text { Sólidos Solúveis } \\
{ }^{\circ} \mathrm{BRIX}\end{array}$ & Vitamina C \\
\hline $25^{\circ} \mathrm{C} / 1$ hora & $4,07 \pm 0,02^{b}$ & $0,12 \pm 0^{\mathrm{a}}$ & $2,45 \pm 0,07^{b}$ & $10,74 \pm 0,24^{b}$ \\
\hline $45^{\circ} \mathrm{C} / 1$ hora & $4,72 \pm 0,32^{\mathrm{ab}}$ & $0,13 \pm 0^{\mathrm{a}}$ & $3 \pm 0^{\mathrm{a}}$ & $7,04 \pm 0^{c}$ \\
\hline $65^{\circ} \mathrm{C} / 1$ hora & $5,24 \pm 0,04^{\mathrm{a}}$ & $0,2 \pm 0,1^{\mathrm{b}}$ & $3,1 \pm 0,14^{a}$ & $21,28 \pm 0^{a}$ \\
\hline $25^{\circ} \mathrm{C} / 12$ horas & $3,96 \pm 0,04^{b}$ & $0,15 \pm 0,01^{b}$ & $2,6 \pm 0^{a}$ & $7,22 \pm 0^{c}$ \\
\hline $45^{\circ} \mathrm{C} / 12$ horas & $4,2 \pm 0,08^{b}$ & $0,14 \pm 0^{\mathrm{a}}$ & $3,1 \pm 0,14^{\mathrm{a}}$ & $10,03 \pm 0,74^{b}$ \\
\hline $65^{\circ} \mathrm{C} / 12$ horas & $5,45 \pm 0,35^{a}$ & $0,23 \pm 0,2^{\mathrm{a}}$ & $3,15 \pm 0,21^{\mathrm{a}}$ & $14,08 \pm 0^{\mathrm{a}}$ \\
\hline $25^{\circ} \mathrm{C} / 24$ horas & $3,95 \pm 0,06^{c}$ & $0,2 \pm 0,2^{a}$ & $2,35 \pm 0,07^{c}$ & $10,74 \pm 0,24^{b}$ \\
\hline $45^{\circ} \mathrm{C} / 24$ horas & $4,5 \pm 0,02^{b}$ & $0,15 \pm 0,02^{\mathrm{ab}}$ & $3 \pm 0^{\mathrm{b}}$ & $10,56 \pm 0^{\mathrm{b}}$ \\
\hline $65^{\circ} \mathrm{C} / 24$ horas & $5,52 \pm 0,01^{\mathrm{a}}$ & $0,17 \pm 0,13^{\mathrm{a}}$ & $4,05 \pm 0,07^{a}$ & $13,73 \pm 0,49^{a}$ \\
\hline \multicolumn{5}{|c|}{ Tocantins } \\
\hline Temperatura & $\mathrm{pH}$ & $\begin{array}{c}\text { Acidez } \\
\text { Titulável Total }\end{array}$ & $\begin{array}{c}\text { Sólidos Solúveis } \\
{ }^{\circ B R I X}\end{array}$ & Vitamina C \\
\hline $25^{\circ} \mathrm{C} / 1$ hora & $4,36 \pm 0,06^{c}$ & $0,22 \pm 0,12^{b}$ & $1,05 \pm 0,07^{c}$ & $10,21 \pm 2,49^{b}$ \\
\hline
\end{tabular}

ENCICLOPÉDIA BIOSFERA, Centro Científico Conhecer - Goiânia, v.14 n.25; p. 18042017 


\begin{tabular}{ccccc}
\hline $45^{\circ} \mathrm{C} / 1$ hora & $4,81 \pm 0,07^{\mathrm{b}}$ & $0,12 \pm 0^{\mathrm{a}}$ & $4,5 \pm 0^{\mathrm{b}}$ & $10,74 \pm 0,24^{\mathrm{b}}$ \\
$65^{\circ} \mathrm{C} / 1$ hora & $5,45 \pm 0,08^{\mathrm{a}}$ & $0,16 \pm 0,3^{\mathrm{c}}$ & $1,95 \pm 0,07^{\mathrm{a}}$ & $25,54 \pm 1,5^{\mathrm{a}}$ \\
$25^{\circ} \mathrm{C} / 12$ horas & $3,99 \pm 0,11^{\mathrm{b}}$ & $0,19 \pm 0^{\mathrm{a}}$ & $1 \pm 0^{\mathrm{b}}$ & $7,044 \pm 0^{\mathrm{b}}$ \\
$45^{\circ} \mathrm{C} / 12$ horas & $4,35 \pm 0,24^{\mathrm{b}}$ & $0,13 \pm 0^{\mathrm{b}}$ & $4,25 \pm 0,35^{\mathrm{a}}$ & $7,04 \pm 0^{\mathrm{b}}$ \\
$65^{\circ} \mathrm{C} / 12$ horas & $5,27 \pm 0,014^{\mathrm{a}}$ & $0,2 \pm 0,4^{\mathrm{a}}$ & $1,5 \pm 0^{\mathrm{b}}$ & $17,08 \pm 0,74^{\mathrm{a}}$ \\
$25^{\circ} \mathrm{C} / 24$ horas & $3,95 \pm 0,05^{\mathrm{a}}$ & $0,18 \pm 0,3^{\mathrm{a}}$ & $1 \pm 0^{\mathrm{c}}$ & $7,04 \pm 0^{\mathrm{b}}$ \\
$45^{\circ} \mathrm{C} / 2$ horas & $4,31 \pm 0,10^{\mathrm{a}}$ & $0,14 \pm 0^{\mathrm{b}}$ & $4 \pm 0^{\mathrm{b}}$ & $7,04 \pm 0^{\mathrm{b}}$ \\
$65^{\circ} \mathrm{C} / 2$ horas & $4,84 \pm 0,91^{\mathrm{a}}$ & $0,1 \pm 0^{\mathrm{c}}$ & $3,5 \pm 0^{\mathrm{a}}$ & $10,56 \pm 0^{\mathrm{a}}$ \\
\hline
\end{tabular}

*Médias seguidas pela mesma letra nas colunas não diferem estatisticamente entre si, pelo teste Tukey, a nível de $5 \%$ de significância.

TABELA 4 - Resultados das análises físico-químicas das extrações alcoólicas do pequi das regiões de Goiás, Minas Gerais e Tocantins.

\begin{tabular}{|c|c|c|c|c|}
\hline \multicolumn{5}{|c|}{ Goiás } \\
\hline Temperatura & $\mathrm{pH}$ & $\begin{array}{c}\text { Acidez } \\
\text { Titulável Total }\end{array}$ & $\begin{array}{c}\text { Sólidos } \\
\text { Solúveis }{ }^{\circ} \text { Brix }\end{array}$ & Vitamina C \\
\hline $25^{\circ} \mathrm{C} / 1$ hora & $6,41 \pm 0,03^{a}$ & $0,1 \pm 0^{\mathrm{a}}$ & $21,3 \pm 0,14^{\mathrm{a}}$ & $10,56 \pm 0^{a}$ \\
\hline $45^{\circ} \mathrm{C} / 1$ hora & $6,43 \pm 0,01^{a}$ & $0,13 \pm 0,12^{a}$ & $20,1 \pm 0,14^{b}$ & $7,04 \pm 0^{b}$ \\
\hline $65^{\circ} \mathrm{C} / 1$ hora & $6,44 \pm 0,01^{a}$ & $0,14 \pm 0^{a}$ & $21,1 \pm 0,14^{\mathrm{a}}$ & $9,86 \pm 0,9^{a}$ \\
\hline $25^{\circ} \mathrm{C} / 12$ horas & $6,44 \pm 0,04^{a}$ & $0,11 \pm 0,1^{\mathrm{a}}$ & $21,25 \pm 0,07^{a}$ & $7,04 \pm 0^{\mathrm{a}}$ \\
\hline $45^{\circ} \mathrm{C} / 12$ horas & $6,34 \pm 0,01^{a b}$ & $0,17 \pm 0,01^{b}$ & $21 \pm 0^{a}$ & $7,04 \pm 0^{a}$ \\
\hline $65^{\circ} \mathrm{C} / 12$ horas & $6,23 \pm 0,04^{b}$ & $0,21 \pm 0^{c}$ & $21,1 \pm 0,14^{a}$ & $7,04 \pm 0^{a}$ \\
\hline $25^{\circ} \mathrm{C} / 24$ horas & $6,32 \pm 0,06^{a}$ & $0,2 \pm 0,2^{a}$ & $21,5 \pm 0^{a}$ & $7,04 \pm 0^{b}$ \\
\hline $45^{\circ} \mathrm{C} / 24$ horas & $6,37 \pm 0,05^{a}$ & $0,17 \pm 0^{a}$ & $20,8 \pm 0,56^{a}$ & $10,56 \pm 0^{a}$ \\
\hline $65^{\circ} \mathrm{C} / 24$ horas & $6,07 \pm 0,03^{b}$ & $0,2 \pm 0^{a}$ & $20,25 \pm 0,35^{a}$ & $7,04 \pm 0^{b}$ \\
\hline \multicolumn{5}{|c|}{ Minas Gerais } \\
\hline Temperatura & $\mathrm{pH}$ & $\begin{array}{c}\text { Acidez } \\
\text { Titulável Total }\end{array}$ & $\begin{array}{c}\text { Sólidos } \\
\text { Solúveis }{ }^{\circ} \text { Brix }\end{array}$ & Vitamina C \\
\hline $25^{\circ} \mathrm{C} / 1$ hora & $6,51 \pm 0,09^{a}$ & $0,15 \pm 0,02^{a}$ & $20,25 \pm 0,07^{b}$ & $10,56 \pm 0^{a}$ \\
\hline $45^{\circ} \mathrm{C} / 1$ hora & $6,63 \pm 0^{a}$ & $0,16 \pm 0^{a}$ & $20 \pm 0^{c}$ & $7,04 \pm 0^{b}$ \\
\hline $65^{\circ} \mathrm{C} / 1$ hora & $6,46 \pm 0,07^{a}$ & $0,14 \pm 0^{a}$ & $21 \pm 0^{a}$ & $7,04 \pm 0^{b}$ \\
\hline $25^{\circ} \mathrm{C} / 12$ horas & $6,53 \pm 0,04^{a}$ & $0,12 \pm 0,1^{a}$ & $20 \pm 0^{\mathrm{a}}$ & $7,04 \pm 0^{a}$ \\
\hline $45^{\circ} \mathrm{C} / 12$ horas & $6,54 \pm 0,01^{a}$ & $0,15 \pm 0^{a}$ & $20,1 \pm 0,14^{a}$ & $7,04 \pm 0^{a}$ \\
\hline $65^{\circ} \mathrm{C} / 12$ horas & $6,37 \pm 0,04^{a}$ & $0,13 \pm 0,01^{a}$ & $20,4 \pm 0,14^{a}$ & $7,04 \pm 0^{a}$ \\
\hline $25^{\circ} \mathrm{C} / 24$ horas & $6,54 \pm 0,04^{a}$ & $0,2 \pm 0,01^{a}$ & $20,15 \pm 0,07^{b}$ & $7,04 \pm 0^{a}$ \\
\hline $45^{\circ} \mathrm{C} / 24$ horas & $6,31 \pm 0,01^{b}$ & $0,24 \pm, 1^{a}$ & $20,3 \pm 0^{b}$ & $7,04 \pm 0^{\mathrm{a}}$ \\
\hline $65^{\circ} \mathrm{C} / 24$ horas & $6,29 \pm 0,02^{b}$ & $0,22 \pm 0^{a}$ & $21 \pm 0^{a}$ & $6,69 \pm 0,49^{a}$ \\
\hline
\end{tabular}
$6{ }^{\circ} \mathrm{C} / 24$ horas $\quad 6,29 \pm 0,02^{\mathrm{b}} \quad 0,22 \pm 0^{\mathrm{a}}$
Tocantins

\begin{tabular}{ccccc}
\hline Temperatura & $\mathrm{pH}$ & $\begin{array}{c}\text { Acidez } \\
\text { Titulável Total }\end{array}$ & $\begin{array}{c}\text { Sólidos } \\
\text { Solúveis }\end{array}{ }^{\circ}$ Brix & Vitamina C \\
\hline $25^{\circ} \mathrm{C} / 1$ hora & $6,65 \pm 0,02^{\mathrm{a}}$ & $0,2 \pm 0,3^{\mathrm{a}}$ & $20 \pm 0^{\mathrm{b}}$ & $7,04 \pm 0^{\mathrm{b}}$ \\
$45^{\circ} \mathrm{C} / 1$ hora & $6,43 \pm 0,04^{\mathrm{b}}$ & $0,2 \pm 0,1^{\mathrm{a}}$ & $20,25 \pm 0,07^{\mathrm{a}}$ & $7,04 \pm 0^{\mathrm{b}}$ \\
$65^{\circ} \mathrm{C} / 1$ hora & $6,47 \pm 0,04^{\mathrm{b}}$ & $0,2 \pm 0^{\mathrm{a}}$ & $20 \pm 0^{\mathrm{b}}$ & $10,56 \pm 0^{\mathrm{a}}$ \\
$25^{\circ} \mathrm{C} / 12$ horas & $6,57 \pm 0,10^{\mathrm{a}}$ & $0,1 \pm 0,1^{\mathrm{a}}$ & $20,1 \pm 0,14^{\mathrm{a}}$ & $3,522 \pm 0^{\mathrm{c}}$ \\
$45^{\circ} \mathrm{C} / 12$ horas & $6,49 \pm 0,02^{\mathrm{a}}$ & $0,1 \pm 0,01^{\mathrm{a}}$ & $20,15 \pm 0,07^{\mathrm{a}}$ & $7,04 \pm 0^{\mathrm{b}}$ \\
$65^{\circ} \mathrm{C} / 12$ horas & $6,29 \pm 0,09^{\mathrm{a}}$ & $0,1 \pm \pm 0^{\mathrm{a}}$ & $19,5 \pm 0,7^{\mathrm{a}}$ & $10,56 \pm 0^{\mathrm{a}}$ \\
\hline
\end{tabular}




\begin{tabular}{lcccc}
\hline $25^{\circ} \mathrm{C} / 24$ horas & $6,5 \pm 0,02^{\mathrm{a}}$ & $0,11 \pm 0,2^{\mathrm{a}}$ & $20 \pm 0^{\mathrm{b}}$ & $10,56 \pm 0^{\mathrm{b}}$ \\
$45^{\circ} \mathrm{C} / 24$ horas & $6,44 \pm 0,01^{\mathrm{a}}$ & $0,2 \pm 0^{\mathrm{a}}$ & $20,5 \pm 0^{\mathrm{a}}$ & $14,08 \pm 0^{\mathrm{a}}$ \\
$65^{\circ} \mathrm{C} / 24$ horas & $6,21 \pm 0^{\mathrm{b}}$ & $0,23 \pm 0,1^{\mathrm{a}}$ & $20 \pm 0^{\mathrm{b}}$ & $7,04 \pm 0^{\mathrm{c}}$ \\
\hline
\end{tabular}

*Médias seguidas pela mesma letra nas colunas não diferem estatisticamente entre si, pelo teste Tukey, a nível de 5\% de significância.

$\mathrm{O} \mathrm{pH}$ dos extratos aquosos de pequi apresentou tendência ao aumento, em função da elevação da temperatura. No entanto, valores esses próximos dos encontrados por PEREIRA (2008), ou seja, 4,9 para extração aquosa de própolis. Já nos extratos alcoólicos os resultados mostraram uma ligeira queda do $\mathrm{pH}$. LONGHINI et al. (2007), encontraram valores de pH 6,35 nos extratos alcoólicos de própolis.

Os resultados obtidos na acidez titulável foram superiores aos obtidos por PINEDO et al. (2010) quer dizer, 0,042\%, estudando a polpa de pequi in natura para verificar a estabilidade da pasta de pequi. Comportamento semelhante foi observado por SOUZA et al., (2007), que verificaram valores médios de acidez titulável de $0,049 \%$ analisando a qualidade de pequi minimamente processado.

Os maiores teores de sólidos solúveis observados foram nos extratos alcoólicos, apresentaram-se altos comparados com os resultados dos extratos aquosos. Ressaltando, que quanto maior a quantidade de sólidos solúveis, menor será a quantidade de sólido a ser adicionada no processo pela indústria. Deste modo, diminuindo o custo de produção, menor tempo de evaporação da água, menor gasto de energia e maior rendimento do produto, resultando em maior economia no processamento (COSTA et al., 2004).

Considerando a recomendação de vitamina $\mathrm{C}$, verificou-se o pequi como uma boa fonte para o suprimento das recomendações diárias. SANTOS et al. (2004), encontraram $78,7 \mathrm{mg} / 100 \mathrm{~g}$, concentrações essas superiores às encontradas na presente pesquisa. Em contrapartida, ao comparar os teores de vitamina $C$ do pequi com outro fruto do cerrado, o jenipapo $(2,7 \mathrm{mg} / 100 \mathrm{~g})$, observou-se que o pequi apresentou maior concentração dessa vitamina C.

\section{Análises físico-químicas dos compostos fenólicos totais e carotenoides totais das extrações aquosas e alcoólicas do pequi}

Nas Figuras 2 e 3 estão os valores de carotenoides totais e de fenólicos totais. Nesta etapa foi realizado um estudo para obter a melhor categoria de extração, utilizando água e álcool etílico como solventes e diferentes temperaturas e tempos de extração. 

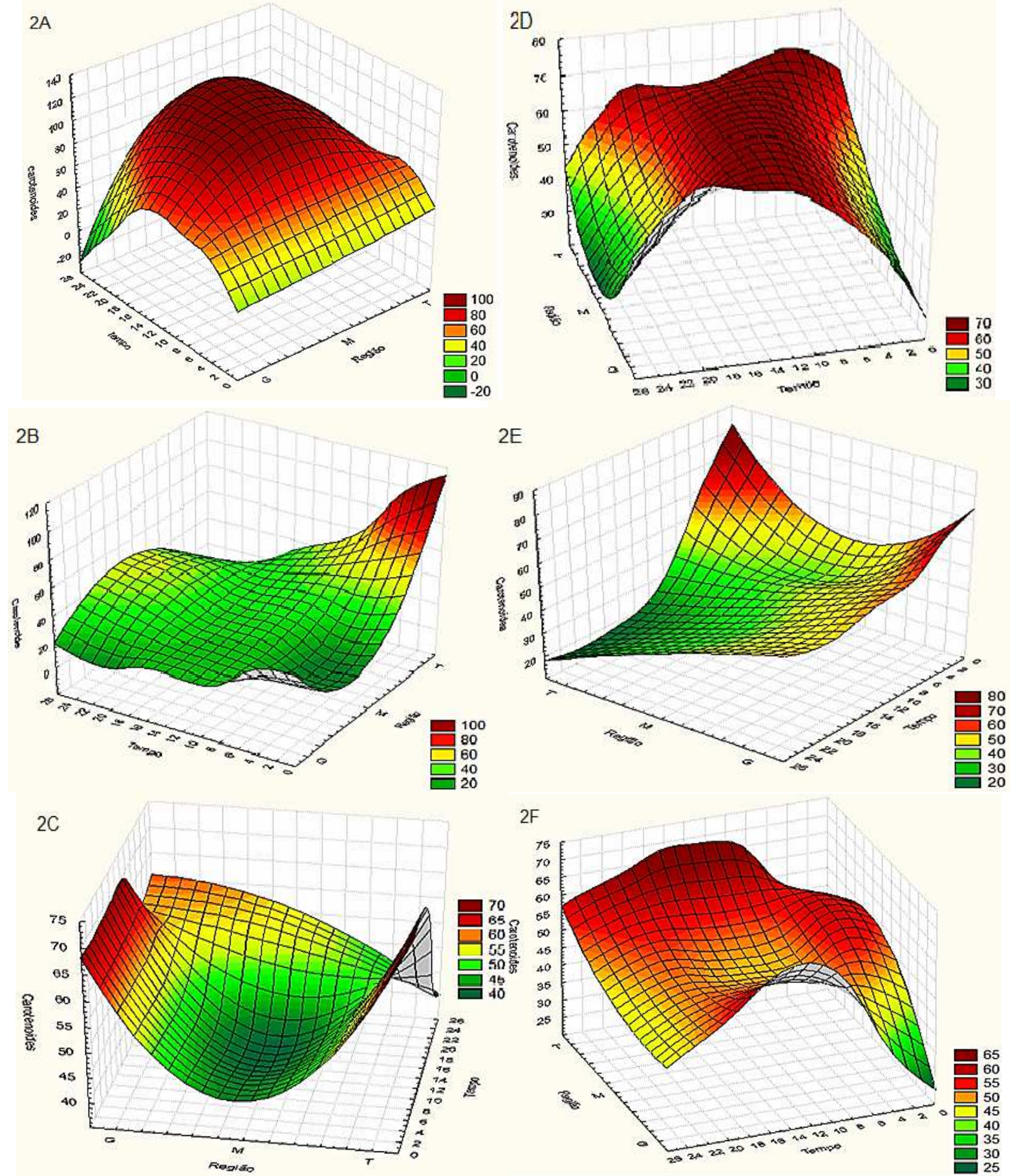

FIGURA 2. $2 \mathrm{~A}, 2 \mathrm{~B}$ e $2 \mathrm{C}$ teores de carotenoides dos extratos aquosos a 25,45 e 65 ${ }^{\circ} \mathrm{C}$ respectivamente, a 1,12 e 24 horas. $2 \mathrm{D}, 2 \mathrm{E}$ e $2 \mathrm{~F}$ teores de carotenoides dos extratos alcoólicos a 25,45 e $65^{\circ} \mathrm{C}$ respectivamente, a 1, 12 e 24 horas. Regiões: $\mathrm{G}=$ Goiás/ $M=$ Minas Gerais/ T=Tocantins 

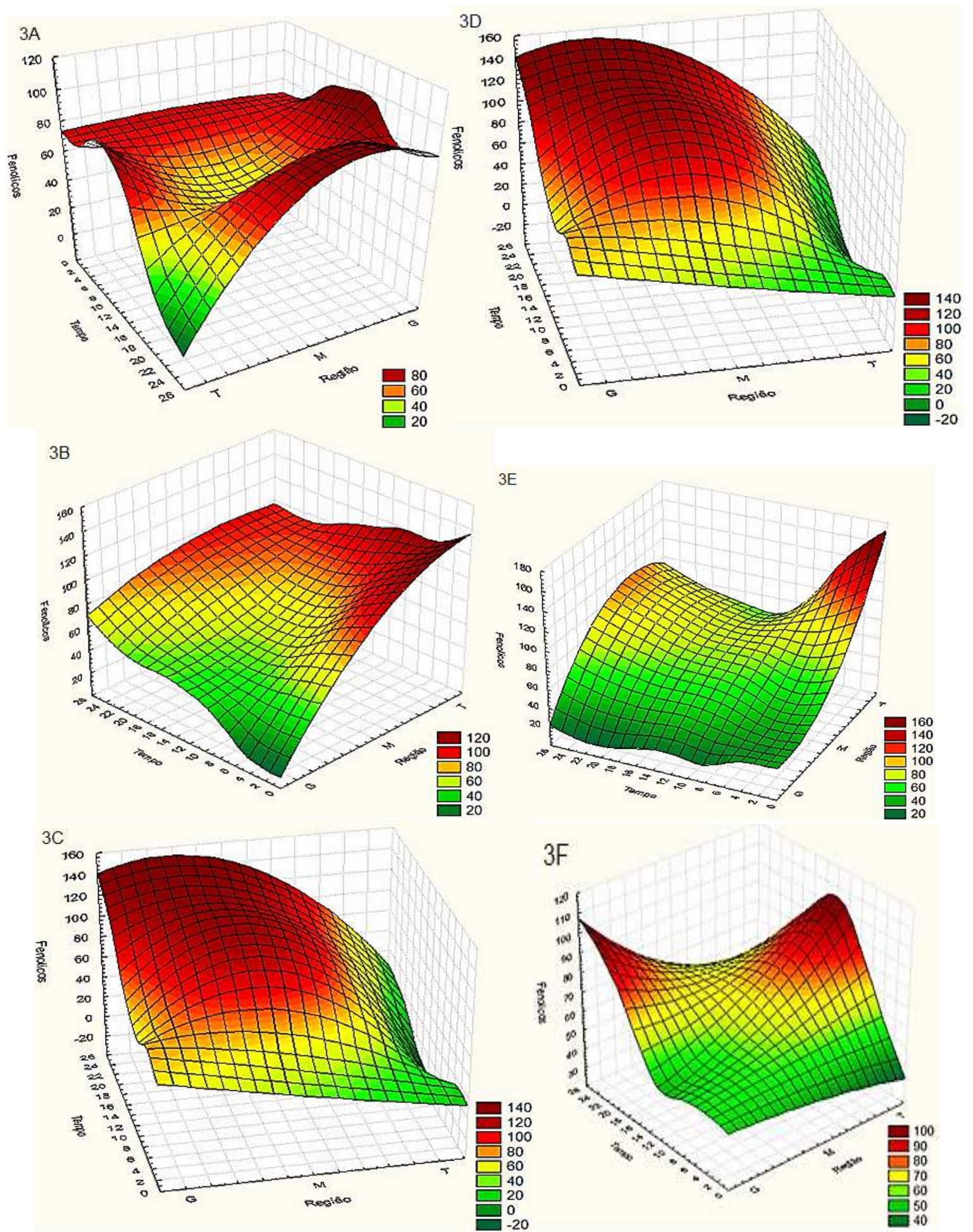

FIGURA 3. 3A, 3B e $3 \mathrm{C}$ teores de fenólicos Totais dos extratos aquosos a 25, 45 e $65{ }^{\circ} \mathrm{C}$ respectivamente, a 1,12 e 24 horas. 3D, 3E e $3 \mathrm{~F}$ teores de fenólicos Totais dos extratos alcoólicos a 25,45 e $65{ }^{\circ} \mathrm{C}$ respectivamente, a 1, 12 e 24 horas. Regiões: $G=$ Goiás/ M= Minas Gerais/ T=Tocantins. 
As variantes influenciaram as duas respostas, sendo que as maiores extrações de carotenoides foram de $80,41 \mathrm{mg} / \mathrm{mL} 12 \mathrm{~h}$ a $25^{\circ} \mathrm{C}$ Goiás, $99,91 \mathrm{mg} / \mathrm{mL}$ $24 \mathrm{~h}$ a $25^{\circ} \mathrm{C}$ Minas Gerais e $86,77 \mathrm{mg} / \mathrm{mL} 1 \mathrm{~h}$ a $45^{\circ} \mathrm{C}$ para Tocantins nos extratos aquosos. E nos extratos alcoólicos consistiu em $68,05 \mathrm{mg} / \mathrm{ml}$ Goiás, $69,84 \mathrm{mg} / \mathrm{mL}$ Minas Gerais e $68,65 \mathrm{mg} / \mathrm{mL} 1 \mathrm{~h} 45^{\circ} \mathrm{C}$ para Tocantins. Observando que a extração aquosa, obteve-se maiores valores de carotenoides totais, assim, como uma alternativa, a água tem se mostrado muito eficiente para extração, sendo benéfica por não apresentar sabor residual. Segundo Hinneburg, Damien e Raimo (2006), a importância dos carotenoides, independente da sua atividade como provitamina $A$, é devido as propriedades antioxidantes. A ingestão de alimentos ricos nesse constituinte tem sido relacionada com o fortalecimento do sistema imunológico e a redução do risco de doenças degenerativas como o câncer.

$\mathrm{Na}$ determinação dos compostos fenólicos totais, que teve maior extração foram Goiás $141,11 \mathrm{mgAG} / \mathrm{L} 24 \mathrm{~h}$ a $65^{\circ} \mathrm{C}$, Minas Gerais $130,51 \mathrm{mgAG} / \mathrm{L} 24 \mathrm{~h}$ a $65^{\circ} \mathrm{C}$ e Tocantins $129,44 \mathrm{mgAG} / \mathrm{L} 1 \mathrm{~h}$ a $45^{\circ} \mathrm{C}$ nos extratos aquosos. $\mathrm{E}$ para os extratos alcóolicos foram Goiás 95,20 mgAG/L 24h a $65^{\circ} \mathrm{C}$, Minas Gerais 97,99 mgAG/L 12h a $25^{\circ} \mathrm{C}$ e para Tocantins $142,83 \mathrm{mgAG} / \mathrm{L} 1 \mathrm{~h}$ a $45^{\circ} \mathrm{C}$. Constatando que, os teores de fenólicos foram superiores nas extrações aquosas, este fato pode ser explicado pelas substâncias fenólicas serem, em sua maioria, hidrossolúveis, ou seja, solúveis em água.

\section{CONCLUSÕES}

As características biométricas foram as que mais apresentaram variabilidade, constatando variações para massa da casca sendo que os frutos de Tocantins foi que apresentou o maior valor.

Nas análises físico-químicas da polpa de pequi as maiores diferenças foram em relação a vitamina $C$, que para Minas Gerais destacou, mostrando de grande importância, pois o ácido ascórbico apresenta atividade biológica.

Para os extratos aquosos e alcoólicos verificou-se que os compostos fenólicos extraídos por solventes de diferentes polaridades refletem resultados de teores diferentes, de modo que se encontre a melhor alternativa para a aplicação em alimentos, e quando se obtém uma extração com teores positivos, com a água, remete-se a um dado animador por ser a água o solvente universal, que está envolvido nos processos culinários e é utilizado como fonte direta de consumo.

Portanto, a perspectiva de se estudar os compostos bioativos do pequi visa a identificação da presença de compostos com atividade antioxidante como os compostos fenólicos e carotenoides, com a perspectiva de uma melhor utilização do pequi, do ponto de vista tecnológico e nutricional, agregando valor ao fruto e aos seus produtos.

\section{REFERÊNCIAS}

BEUCHLEA, R.; GRECCHI, R. C.; SHIMABUKURO, Y. E.; SELIGER, R.; EVA, H. D.; SANO, E.; ACHARD, F. Land cover changes in the Brazilian Cerrado and Caatinga biomes from 1990 to 2010 based on a systematic remote sensing sampling approach. Applied Geography, v. 58, n.2, p. 116-127, 2015. Disponível em http://dx.doi.org/10.1016/j.apgeog.2015.01.017. doi 10.1016/ S0143622815000284

COSTA, W. S.; FILHO, J. S.; EDUARDO, M. MATA, C.; QUEIROZ, A. J. M. Influência da concentração de sólidos solúveis totais no sinal fotoacústico de polpa de manga. Revista Brasileira de Produtos Agroindustriais, Campina Grande, v.6, 
n.2, p.141-147, 2004.

DANTAS, R.L.; ROCHA, A. P. T.; ARAÚJO, A. S.; RODRIGUES, M. S.; MARANHÃO, T. K. L. Perfil da qualidade de polpa de frutas comercializadas na cidade de Campina Grande /PB. Revista Verde de Agroecologia e Desenvolvimento Sustentável, Mossoró, v.5, n.5, p.61-66, 2010.

FERREIRA, G. A.; NAVES, R. V.; CHAVES, L. J.; VELOSO, V. R.; SOUZA, E. R. B. Produção de frutos de populações naturais de pequizeiro no estado de goiás. Revista Brasileira Fruticultura, Jaboticabal, v. 37, n. 1, p. 121-129, 2015.

HINNEBURG, I; DAMIEN, H. J; RAIMO H. Antioxidant activities of extracts from selected culinary herbs and spices. Food Chemistry, London, v. 97, n. 1, p. 122129, 2006.

IAL - Instituto Adolfo Lutz. Normas Analíticas do Instituto Adolfo Lutz. Métodos químicos e físicos para análise de alimentos. v. 1, 5. ed. São Paulo: Instituto Adolfo Lutz, 2008.

LEÃO, D. P.; FRANCA, A. S.; OLIVEIRA, L. S.; BASTOS, R.; COIMBRA, M. A. Caracterização físico-química, a capacidade antioxidante, fenólico total, o teor de proantocianidinas da farinha preparados a partir de pequi ( Caryocar brasilense Camb.) Fruta subprodutos. Food Chemistry, v. 225, n. 15, p. 146-153, 2017. Disponível em http://dx.doi.org/10.1016/j.foodchem.2017.01.027. doi 10.1016/ S0308814617300262

LIMA, A. SILVA, A. M. O.; TRINDADE, R. A.; TORRES, R. P.; MANCINI-FILHO, J. Composição química e compostos bioativos presentes na polpa e na amêndoa do pequi (Caryocar brasiliense Camb.). Revista Brasileira de Fruticultura, Jaboticabal, v. 29, n. 3, p. 695-698, 2007.

LONGHINI, R.; RAKSA, S. M.; OLIVEIRA, A. C.; SVIDZINSKI, T. I. E.; FRANCO, S. L. Obtenção de extratos de própolis sob diferentes condições e avaliação de sua atividade antifúngica. Revista Brasileira Farmacognosia Brazilian Journal of Pharmacognosy, Maringá, v.17, n.3, p. 388-395, 2007. Disponível em http://dx.doi.org/10.1590. doi 10.1590/S0102-695X2007000300015

MACHADO, M. T. C.; MELLO, B. C. B. S.; HUBINGER, M. D. Evaluation of pequi (Caryocar Brasiliense Camb.) aqueous extract quality processed by membranes. Food and Bioproducts Processing, v. 95, n. 2, p. 304-312, 2015. Disponível em http://dx.doi.org/10.1016/j.fbp.2014.10.013. doi 10.1016/S0960308514001345

MACHADO, M. T. C.; MELLO, B. C. B. S.; HUBINGER. Study of alcoholic and aqueous extraction of pequi (Caryocar brasiliense Camb.) natural antioxidants and extracts concentration by nanofiltration. Journal of Food Engineering, v. 117, n. 6, p. 450-457, 2013. Disponível em http://dx.doi.org/10.1016/j.jfoodeng.2012.12.007. doi 10.1016/ S0260877412005912 
MENDONÇA, K. S.; CORRÊA, J. L. G.; JUNQUEIRA, J. R. J.; CIRILLO, M. A.; FIGUEIRA, F. V.; CARVALHO, E. E. N. Influences of convective and vacuum drying on the quality attributes of osmo-dried pequi (Caryocar brasiliense Camb.) slices. Food Chemistry, v. 224, n. 3, p. 212-218, 2017.

MOURA, N. F.; CHAVES, L. J.; NAVES, R. V. Caracterização física de frutos de pequizeiro (caryocar brasiliense camb.) do cerrado. Revista Árvore, Viçosa, v. 37, n. 5, p. 905-912, 2013.

MOURA, N. F.; CHAVES, L. J.; NAVES, R. V. Caracterização física de frutos de pequizeiro (Caryocar brasiliense Camb.) do cerrado. Revista Árvore, v. 37, n. 5, p. 906-912, 2013.

OLIVEIRA, M. E. B.; GUERRA, N. B.; MAIA, A. H. N.; ALVES, R. E.; MATOS, N. A. S.; SAMPAIO, F. G. M.; LOPES, M. M. T. Características químicas e físico-químicas de pequis da Chapada do Araripe, Ceará. Revista Brasileira de Fruticultura, Jaboticabal, v. 32, n. 1, p. 114-125, 2010.

PAZ, J. G.; PACHECO, P.; SILVA, C. O.; PASCOAL, G. B. Análise da composição nutricional e de parâmetros físico-químicos do pequi (caryocar brasiliense camb) in natura. Revista Científica Linkania Master, Uberlândia, v. 1, n. 5, p. 86-159, 2014.

PEREIRA, L.M.; RODRIGUES, A. C. C.; SARANTÓPOULOS, C. I. G. L.; JUNQUEIRA, V. C. A.; CARDELLO, H. M. A B.; HUBINGER, M. D. Vida de prateleira de goiabas minimamente processadas adicionadas em embalagens sob atmosfera modificada. Revista Ciência e Tecnologia de Alimentos, Campinas, v. 23, n. 3, p. 427- 433, 2003.

PESSOA, A. S.; PODESTÁ, R.; BLOCK, J. M.; FRANCESCHI, E.; DARIVAC, C.; LANZAA, M. Extraction of pequi (Caryocar coriaceum) pulp oil using subcritical propane: Determination of process yield and fatty acid profile. The Journal of Supercritical Fluids, v. 101, n. 3, p. 95-103, 2015.

PINEDO, A. A.; MACIEL, V. B. V.; CARVALHO, K. M.; COELHO, A. F. S.; GIRALDOZUNIGA, A. D.; AREVALO, Z. D. S.; ALVIM, T. C. Processamento e estudo da estabilidade de pasta de pequi (Caryocar brasiliense). Revista Ciência e Tecnologia de Alimentos, Campinas, v.30, n.3, 2010.

RAMOS, K. M. C., SOUZA, V. A. B. Características físicas e químico-nutricionais de frutos de pequizeiro (caryocar coriaceum wittm.) em populações naturais da região meio-norte do brasil. Revista Brasileira de Fruticultura, Jaboticabal, v. 33, n. 2, p. 500-508, 2011.

RESENDE-MOREIRA, L. C.; VASCONCELOS, P. N.; SOUTO, A. P.; MENEZES, A. P. A.; LEMOS-FILHO, J. P.; LOVATO, M. B. East-west divergence in central Brazilian Cerrado revealed by cpDNA sequences of a bird-dispersed tree species. Biochemical Systematics and Ecology, v. 70, n. 6, p. 247-253, 2017. Dispinível em http://dx.doi.org/10.1016/j.bse.2016.12.007. doi: 10.1016 
ROCHA, M. S.; FIGUERESO, R. W.; ARAUJO, M. A. M.; MOREIRA-ARAUJO, R. S. R. Caracterização físico-química e atividade antioxidante (in vitro) de frutos do cerrado Piauiense. Revista Brasileira de Fruticultura, Jaboticabal, v. 35, n. 4, p. 933-941, 2013.

ROCHA, W. S.; LOPES, R. M.; SILVA, D, B.; VIEIRA, R. F.; SILVA, J. P.; AGOSTINICOSTA, T. S. Compostos fenólicos totais e taninos condensados em frutas nativas do cerrado. Revista Brasileira Fruticultura, Jaboticabal, v. 33, n. 4, p. 1215-1221, 2011.

SANTOS, F.A.; JESUS, J. R.; FILHO, C.; NONATO, R. Análise qualitativa das polpas congeladas de frutas produzidas pela SUFRUTS, MA. Revista Higiene Alimentar, São Paulo, v. 18, n. 119, p.14-22, 2004.

SANTOS, P.; PORTO, A. G.; SILVA, F. S.; FURTADO, G. F. Avaliação físico química e sensorial do pequi (caryocar brasiliensis camb.) submetido à desidratação. Revista Brasileira de Produtos Agroindustriais, Campina Grande, v. 12, n. 2, p. 115-123, 2010.

SILVA, C. R. M.; NAVES, M. M. V. Suplementação de vitaminas na prevenção de câncer. Revista de Nutrição, Campinas, v. 1, n2, p.135-143, 2001.

SOUSA, F. C.; SILVA, L. M. M.; SOUSA, E. P.; LIMA, A. K. V. O.; FIGUEIREDO, R. M. F. Parâmetros físicos e físico-químicos da polpa de pequi. Revista Verde, Mossoró, v.6, n.1, p. 12-15, 2012.

SOUSA, M. S. B.; VIEIRA, L. M.; LIMA, A. Fenólicos totais e capacidade antioxidante in vitro de resíduos de polpas de frutas tropicais. Brazilian Journal of Food Technology, v. 14, n. 3, p. 202-210, 2011. Disponível em http://www.scielo.br/pdf/bjft/v14n3/04. doi 10.4260/BJFT2011140300024

SOUZA, E.; BOAS, E. V. B.; BOAS, B. M. V.; RODRIGUES, L. J.; PAILA, N. R. F. Qualidade e vida útil de pequi minimamente processado armazenado sob atmosfera modificada. Revista Ciência Agrotécnica, Lavras, v.31, n. 6, p.1811-1817, 2007.

TORRES, M. P. R.; DOROTEU, K.; VALLADÃO, D. M. S.; GLUSCZAK, L.; AGOSTINI, J. S. Características Físicas e Nutricionais do Pequi e Comparação de Diferentes Métodos para Extração de seus Lipídeos. Journal Scientific Electronic Archives, Sinop, v. 1, n.2, p. 6-10, 2012.

TRAESEL, G. K.; MENEGATI, S. E. L. T.; SANTOS, A. C.; SOUZA, R. I. C.; BOAS, G. R. V.; JUSTI, P. N.; KASSUYA, C. A. L.; ARGANDONA, E. J. S.; OESTERREICH, S. A. Oral acute and subchronic toxicity studies of the oil extracted from pequi (Caryocar brasiliense, Camb.) pulp in rats. Food and Chemical Toxicology, v. 97, n.3, p. 224-231, 2016. Disponível em http://dx.doi.org/10.1016/j.fct.2016.09.018. doi S0278691516303337

VERA, R.; NAVES, R. V.; NASCIMENTO, J. L.; CHAVES, L. J.; LEANDRO, W. M.; SOUZA, E. R. B. Caracterização física de frutos do pequizeiro (Caryocar brasiliense Camb.) no estado de Goiás. Pesquisa Agropecuária Tropical, Goiânia, v. 35, n. 2, 
p- 71-79, 2005.

VERA, R.; SOUZA, E. R. B.; FERNANDES, E. P.; NAVES, R. V.; JUNIOR, M. S. S.; CALIARI, M.; XIMENES, P. A. Caracterização física e química de frutos do pequizeiro (caryocar brasiliense camb.) Oriundos de duas regiões no estado de goiás, brasil. Revista Agropecuária Tropical, Goiânia, v. 37, n. 2, p. 93-99, 2007. 